\title{
Video Article \\ Viral Concentration Determination Through Plaque Assays: Using Traditional and Novel Overlay Systems
}

\author{
Alan Baer ${ }^{1}$, Kylene Kehn-Hall ${ }^{1}$ \\ ${ }^{1}$ National Center for Biodefense and Infectious Diseases, George Mason University
}

Correspondence to: Kylene Kehn-Hall at kkehnhal@gmu.edu

URL: https://www.jove.com/video/52065

DOI: doi:10.3791/52065

Keywords: Virology, Issue 93, Plaque Assay, Virology, Viral Quantification, Cellular Overlays, Agarose, Avicel, Crystal Violet Staining, Serial Dilutions, Rift Valley fever virus, Venezuelan Equine Encephalitis, Influenza

Date Published: $11 / 4 / 2014$

Citation: Baer, A., Kehn-Hall, K. Viral Concentration Determination Through Plaque Assays: Using Traditional and Novel Overlay Systems. J. Vis. Exp. (93), e52065, doi:10.3791/52065 (2014).

\section{Abstract}

Plaque assays remain one of the most accurate methods for the direct quantification of infectious virons and antiviral substances through the counting of discrete plaques (infectious units and cellular dead zones) in cell culture. Here we demonstrate how to perform a basic plaque assay, and how differing overlays and techniques can affect plaque formation and production. Typically solid or semisolid overlay substrates, such as agarose or carboxymethyl cellulose, have been used to restrict viral spread, preventing indiscriminate infection through the liquid growth medium. Immobilized overlays restrict cellular infection to the immediately surrounding monolayer, allowing the formation of discrete countable foci and subsequent plaque formation. To overcome the difficulties inherent in using traditional overlays, a novel liquid overlay utilizing microcrystalline cellulose and carboxymethyl cellulose sodium has been increasingly used as a replacement in the standard plaque assay. Liquid overlay plaque assays can be readily performed in either standard 6 or 12 well plate formats as per traditional techniques and require no special equipment. Due to its liquid state and subsequent ease of application and removal, microculture plate formats may alternatively be utilized as a rapid, accurate and high throughput alternative to larger scale viral titrations. Use of a non heated viscous liquid polymer offers the opportunity to streamline work, conserves reagents, incubator space, and increases operational safety when used in traditional or high containment labs as no reagent heating or glassware are required. Liquid overlays may also prove more sensitive than traditional overlays for certain heat labile viruses.

\section{Video Link}

The video component of this article can be found at https://www.jove.com/video/52065/

\section{Introduction}

The accurate isolation and quantification of viable viral samples has consistently been an ongoing research goal in virology. It was not until the advent of the plaque assay in 1952 that a means to quantitatively and qualitatively calculate animal viral titers was first developed ${ }^{1,2}$. This technique was first adapted and modified from phage assays, which had previously been used to calculate titers of stock bacteriophages in plant biology 1,2 . While alternative means for viral quantification have since been developed and adapted, such as immunoassays, fluorescence and transmission electron microscopy, tunable resistive pulse sensing (TRPS), flow cytometry, recombinant reporter systems, and quantitative reverse transcription polymerase chain reaction (qRT-PCR), these methods fail to identify and quantitate replication competent virons ${ }^{1,3}$. While advances in technologies and techniques continue to refine and alter the landscape; plaque assays continue to represent the gold standard in determining viral concentrations for infectious lytic virons ${ }^{1,4}$.

During a plaque assay, a confluent monolayer of host cells is infected with a lytic virus of an unknown concentration that has been serially diluted to a countable range, typically between 5-100 virions. Infected monolayers are then covered with an immobilizing overlay medium to prevent viral infection from indiscriminately spreading through either the mechanical or convectional flow of the liquid medium during viral propagation. While solid or semisolid overlays such as agarose, methyl cellulose or carboxymethyl cellulose (CMC) have traditionally been used, liquid overlays have become an increasingly attractive alternative with the development of novel liquid overlays such as Avicel ${ }^{5-7}$. Plaque assays utilizing liquid versus traditional overlays have several advantages as the overlay can be applied at room temperature, and application and removal is significantly easier. As liquid overlays do not require warming, delicate and heat labile viruses may also prove easier to plaque.

After the initial infection and application of the immobilizing overlay, individual plaques, or zones of cell death, will begin to develop as viral infection and replication are constrained to the surrounding monolayer. Infected cells will continue the replication-lysis-infection cycle, further propagating the infection, resulting in increasingly distinct and discrete plaques. Depending on the viral growth kinetics and host cell used, a visible plaque will normally form within 2-14 days. Cellular monolayers may then be counted with a standard bright field microscope, or more typically fixed and counterstained by neutral red or crystal violent in order to readily identify plaques with the naked eye. There are a wide variety of plaque counter stains available, each offering their specific advantages and disadvantages. Crystal violet is typically added at the point of collection and after the fixation/removal of the overlay, providing a rapid and distinct counter stain which allows for the identification of very small plaques when mixed morphology is present. Neutral red has the advantage of early application and constant contact with the overlay, allowing for the live monitoring of developing plaque formation, which is particularly useful when working with an unknown virus or replication kinetics. We 
have however found that staining is typically not as distinct when using neutral red. 3-(4,5-dimethylthiazol-2-yl)-2,5-diphenyl tetrazolium bromide (MTT) offers several advantages, as the yellow colored dye stains live cells dark blue and viral plaques can be counted without removal of the overlay as with neutral red. The high contrast between live and dead cells afforded by MTT also permits the detection of small plaques at an earlier time point post infection, although storage would still require removal of the overlay ${ }^{8}$. As crystal violet can be simply made in a solution of water and alcohol, and provides a high degree of sensitivity for mixed plaque morphology, we have chosen it as a preferred and simplified counter stain for the protocol demonstrated as we are utilizing several families of well characterized viruses.

After fixing and staining the infected cellular monolayer, plaques are counted in order to titer viral stock samples in terms of plaque forming units (pfu) per milliliter. A log drop should be noted between serial dilutions and, depending on plate size, between 5-100 plaques counted, with a negative control used as a reference. Statistically samples will vary by $10 \%$ for every 100 plaques counted when comparing sample replicates ${ }^{3}$. The advantage of using plaque assays to determine viral titers lies in their ability to quantitate the actual number of infectious viral particles within the sample. As multiple virions could potentially infect a single cell, the terminology of units versus virons is used during plaque titrations ${ }^{1,2}$.

Plaque morphology can vary dramatically under differing growth conditions and between viral species. Plaque size, clarity, border definition, and distribution should all be noted as they can provide valuable information on the growth and virulence factors of the virus in question.

Basic plaque assay principles can also be adapted and modified in a number of different ways, such as in the use of focus forming assays (FFAs). FFAs do not rely on cell lysis and counterstaining to detect plaque formation, but rather employ immunostaining techniques to directly detect intracellular viral proteins through tagged antibodies. Increased sensitivity, decreased incubation times after infection, and most importantly the ability to quantitate non-lytic viruses are all distinct advantages when employing FFAs. While widely used, the critical limiting factors in FFAs versus a traditional plaque assay lies in the need for appropriate antibodies and the ability to only probe for viral protein subunits versus actual infectious virons ${ }^{4}$

For the purpose of this study, we will limit our discussion to classical plaque assays and describe the use of traditional solid and semisolid overlays (agarose and $\mathrm{CMC}$ ), along with novel liquid microcrystalline cellulose overlays.

\section{Protocol}

\section{Preparation of Cells and Reagents}

1. The day prior to the assay, plate appropriate host cells for the virus in question (Table 1 and 2 ) at $90-100 \%$ confluency.

2. Prepare fixing solution of $10 \%$ formaldehyde in $\mathrm{dH}_{2} \mathrm{O}$. In example, mix $5.56 \mathrm{ml}$ of $36 \%$ stock formaldehyde with $14.44 \mathrm{ml}$ distilled $\mathrm{H}_{2} \mathrm{O}\left(\mathrm{dH} \mathrm{H}_{2} \mathrm{O}\right)$. NOTE: Use appropriate safe handling practices and ventilation when using formaldehyde.

3. Prepare crystal violet stain: $1 \%$ Crystal Violet (CV) in $20 \%$ ethanol and $\mathrm{dH}_{2} \mathrm{O}$.

4. Prepare $2 x$ plaque media (cell type dependent at $2 x$ concentration; see Table 2). Filter using a $0.2 \mu m$ filter if any reagents are not sterile.

5. Preparation of immobilizing overlays (Table 3)

1. For liquid overlays, make a sterile $2.4 \%$ solution of Avicel in $\mathrm{dH}_{2} \mathrm{O}$. To prevent clumping, add the powder to a flask containing water that is rapidly mixing with a stir bar, pour Avicel in slowly and stir rapidly at room temperature (RT). When the solution proves too viscous for a stir bar to homogenize, switch to a flask shaker for $>30$ min with heavy agitation to ensure even homogenization. NOTE: Stock solutions can be made at lower concentrations, but the solutions may separate and will need to be remixed to ensure homogenization. Working solutions of Avicel can be used ranging from $0.6-3 \%$.

2. After homogenization, autoclave the solution and store sealed at RT.

3. For agarose and carboxymethyl cellulose $(\mathrm{CMC})$ overlays, prepare a stock solution in $\mathrm{dH}_{2} \mathrm{O}$ of $2 \% \mathrm{CMC}$ or $0.6 \%$ agarose. Mix with a stir bar and autoclave or microwave to bring into solution.

\section{Dilutions and Infections}

1. The day after plating, visually check the confluency and viability of the cells prior to starting the assay. Ensure standard cellular morphology and a $\sim 90 \%$ confluent monolayer is present.

2. Perform a tenfold serial dilution of the infectious samples. Use the cellular growth media for viral propagation as the diluent (Table 2). Vary the number of dilutions required based on the expected titer of the virus in question, and always use an uninfected control sample to independently ensure cellular viability and aid in plaque identification.

3. From the serial dilutions, infect the cells for $45 \mathrm{~min}$ to $1 \mathrm{hr}$ (Table 1). Use a sufficient volume of inoculum to cover the cells, while keeping the volume as low as possible to maximize viral contact with the monolayer (Table 4). Gently rock plates every 20 min to ensure even coverage and prevent the cellular monolayer from drying.

4. After the infection, overlay an appropriate volume of immobilizing medium directly to the inoculums in the well (Table 2) using a 1:1 mix of $2 x$ plaque media and the immobilizing overlay of choice (CMC, agarose or Avicel). Gently rock to mix.

1. For liquid overlays, mix 1:1 with warmed $2 x$ plaque media and $1.2-2.4 \%$ RT stock Avicel to obtain a working solution of $0.6-1.2 \%$ Avicel overlay medium.

2. For an agarose overlay, use a $1: 1$ mixture of warmed $2 x$ plaque media and a stock solution of heated $0.6 \%$ agarose, place in a $56{ }^{\circ} \mathrm{C}$ water bath for 30 min to equilibrate the temperature obtaining a final agarose/overlay concentration of $0.3 \%$.

3. For CMC, prepare a $2 \%$ stock solution and treat as described for the agarose overlay.

4. When applying the overlay to the monolayer, always equilibrate hot agarose or $\mathrm{CMC}$ in a $56{ }^{\circ} \mathrm{C}$ water to prevent damage to the monolayer. Ensure the solution is warm, but not hot to the touch to prevent cell death and reduced viral titers. Most agarose overlays will begin to solidify below $42{ }^{\circ} \mathrm{C}$, work quickly and/or prepare small batches to prevent solidification while handling. 
5. After addition of the overlay, incubate plates to produce distinct plaques that are clearly countable. Plaque formation can take $2-14$ days depending on the virus being analyzed, Table 1.

NOTE: Once liquid overlaid plates are placed in the incubator, do not move them. Movement of the liquid overlays during the incubation period will result in smeared plaques. Agarose and CMC overlays are semisolid and can be moved or checked periodically under a light microscope to monitor plaque development.

\section{Fixing and Staining Cells}

1. To fix the cells, pour off or aspirate the Avicel overlay, and fix cells using the $10 \%$ formaldehyde solution for $30 \mathrm{~min}$ to overnight $(<1 \mathrm{ml}$ per well for a 6 well plate).

1. For agarose or $\mathrm{CMC}$, directly add the formaldehyde solution to the overlay for $1 \mathrm{hr}$ to overnight.

NOTE: Samples can be kept for extended periods of time in the fixative provided it does not evaporate and dry out as this can distort the monolayer.

2. Prior to staining and after fixation, discard the formaldehyde and remove the semisolid plugs for the agarose and CMC with either running water or manually with a spatula. Rinse Avicel plaques with water to remove residual overlay/fixative prior to staining.

3. For staining, cover the cells with a minimal amount of crystal violet solution for $\sim 15 \mathrm{~min}$. Rock plates if necessary to ensure even coverage.

4. Gently wash off the crystal violet stain with water. Once fixed, stained, and dried, store plaques indefinitely for future analysis.

\section{Determining Viral Titers}

1. Count the plaques in each well, taking the average for any technical replicates of the same dilution. For large plate formats, discount wells with fewer than 5 or greater than 100 plaques. Take note of plaque size and morphology. The negative control should have a uniform monolayer and can be used as a reference control.

2. Determine the viral titer of the stock sample by taking the average number of plaques for a dilution and the inverse of the total dilution factor.

$$
\mathrm{Pfu} / \mathrm{ml}=\frac{\text { Avg. \# of plaques }}{\mathrm{D} \times \mathrm{V}}
$$

$\mathrm{D}=$ dilution $\mathrm{V}=$ volume of diluted virus added to the plate

NOTE: As an example, 30 and 32 plaques counted for replicates of the $1 \times 10^{-7}$ dilution [31 (average)/10 $0^{-7}$ (dilution) $\times 0.4 \mathrm{ml}$ (inoculum)] would yield a titer of $7.75 \times 10^{8} \mathrm{pfu} / \mathrm{ml}$.

\section{Representative Results}

The ability of plaque assays to accurately assess viral titers relies upon numerous factors: appropriate host cell selection, proper media and growth conditions for cellular and viral viability, immobilized viral propagation, and an accurate determination of the viral incubation period to allow adequate time for distinct and countable plaque formation.

For this study, viruses from three representative families were chosen to demonstrate differences in: overlay selection, incubation periods, and plaque morphology across differing sample types. Venezuelan Equine Encephalitis (VEEV) was selected as a (+)ssRNA viral model, which can cause significant disease in equine species and humans and represents the Togaviridae family. Influenza B Taiwan strain, a segmented (-)ssRNA virus primarily infecting humans, represents the Orthomyxoviridae family. Rift Valley fever virus (RVFV), a (-)ssRNA arthropod born virus primarily infecting arthropods, ruminants and humans, was selected as a representative of the Bunyaviridae family.

For RVFV (Figure 1), titers were determined from a stock solution of a recombinant live attenuated MP12 strain of RVFV in a 12 well plate format utilizing $\mathrm{CMC}$, agarose, or Avicel overlays which were incubated side-by-side for $72 \mathrm{hr}$ post infection (hpi). A representative plate showing dilutions ranging from $10^{-4}$ to $10^{-7}$ can be seen in Panel A. Plaques using CMC and agarose overlays showed small, clear, and distinct plaques with a well-defined circular border. Plaques with a liquid overlay were slightly more abundant and larger in comparison to agarose and $\mathrm{CMC}$ plaques, and provided a less distinct border. Viral titers were compared in Panel B with all of the overlays performing comparably.

In order to obtain a clearer visual comparison among overlays for MP12 along with a larger sample size to determine reproducibility, a 6 well plate was also trialed in triplicate (Figure 2). In the 6 well plate format, the use of a CMC overlay showed smaller plaques than either agarose or liquid overlays, which were comparable in size to each other. While viral titers were similar between all three overlays (Panel D), plaques formed in agarose and liquid overlays proved easier to count due to their increased size.

In contrast with RVFV, VEEV titers and plaque morphology among the differing overlays varied markedly (Figure 3A). Plaques formed in CMC overlays demonstrated a clear and distinct morphology when using a 12 well plate format, at the expense of plaque size and sensitivity (Panel B). In contrast to CMC, the use of agarose and liquid overlays resulted in significantly larger plaques, indicating lower viral inhibition and increased sensitivity to VEEV replication. This was previously confirmed when solely comparing agarose versus CMC in a paper published by Juarez et al. ${ }^{4}$. While agarose and liquid overlays produced larger plaques than CMC, the plaques had poorly defined borders and were difficult to count in a 12 well format, with liquid overlays providing the greatest border diffusion. When plaques were trialed in 6 well plates (Figure 4), the larger 6 well format negated the issue of overtly large plaques that were difficult to differentiate in the 12 well format, with agarose and liquid overlays proving superior to the CMC overlays in terms of plaque definition and sensitively (Figure 4D).

In comparison to RVFV or VEEV, influenza provides several unique challenges when plaquing, such as the requirement of an external protease. The sensitivity of influenza virus to differing overlay selections has also been well documented in the past as significant changes have been noted when modifications as minor as differing brands of agarose have been used ${ }^{9}$. 
Interestingly for the Influenza B Taiwan strain, the use of CMC as an overlay resulted in markedly smaller plaques that were difficult to count and proved difficult to reliably score (Figure 5A). The use of an agarose overlay provided the best plaques (Panel C), and resulted in a darker background stain (likely due to increased monolayer viability), and showed clearer and sharper plaques in direct comparison to the use of the liquid overlay (Panel B).

A distinct advantage of liquid polymers over solid and semisolid overlays, such as agarose and CMC, lies in the ease of removal and application. Semisolid overlays require heating, and solidification can prove problematic when handling and removing. In order to capitalize on these advantages and to determine the practicality of utilizing Avicel in a high-throughput manner for RVFV, a 96 well plate format was trialed at varying overlay concentrations (Figure 6). For RVFV MP-12, dilutions were performed in quadruplicate at both 0.6 and $1.2 \%$ final concentrations of Avicel. Overlay application and removal proved simple, with no apparent differences among replicates or between concentrations noted, demonstrating a high degree of reproducibility. When scoring, plaques were distinct and countable to the naked eye, demonstrating the feasibility of utilizing liquid overlays in a high throughput manner for RVFV.

A)

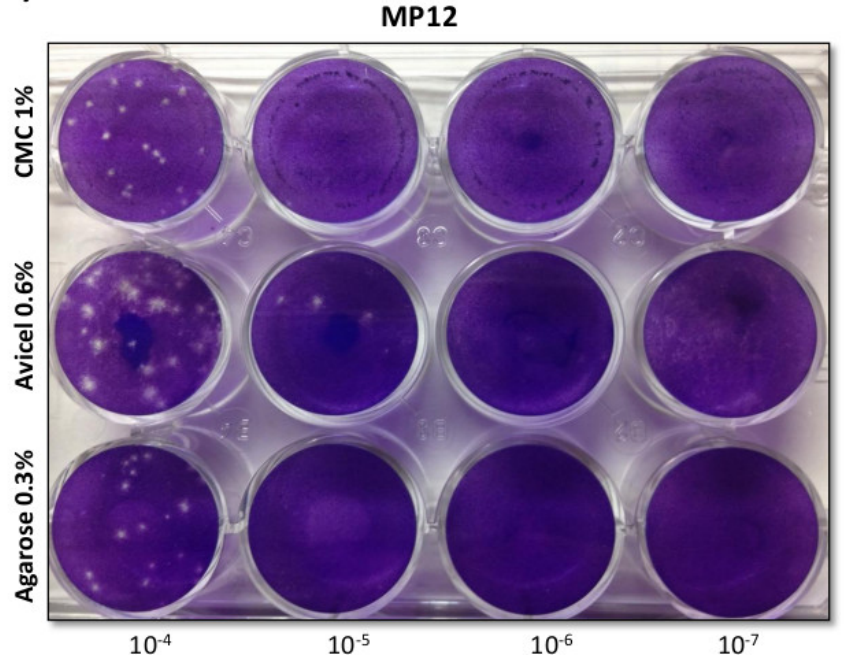

B)

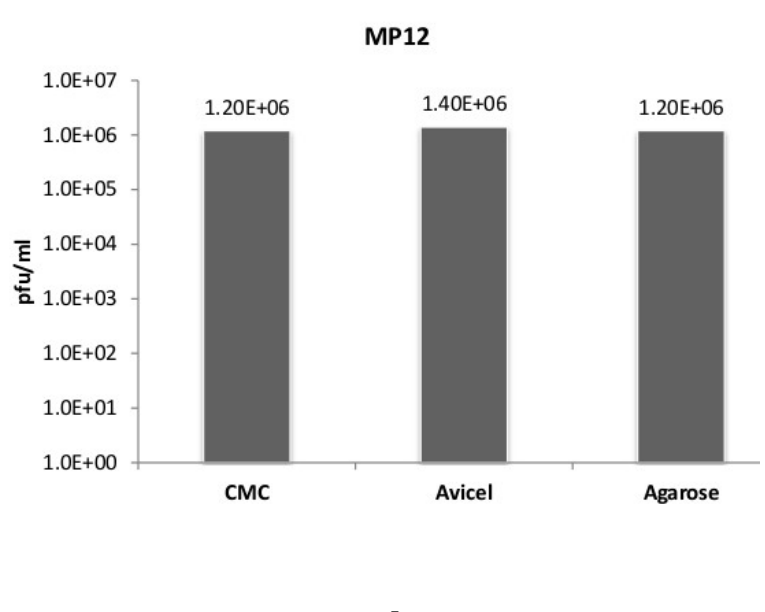

Figure 1: RVFV plaque overlay comparisons utilizing 12 well plates. Veros were plated at $2.5 \times 10^{5}$ cells in 12 well plates and infected with $200 \mu \mathrm{l}$ using the same serially diluted starting sample of MP12. After infection $1.5 \mathrm{ml}$ overlays of $0.3 \%$ agarose, $0.6 \%$ Avicel, or $1 \%$ CMC (final concentrations), were applied in order to directly compare the overlays as demonstrated in Panel A. Plaques were counted and titered in Panel B. 


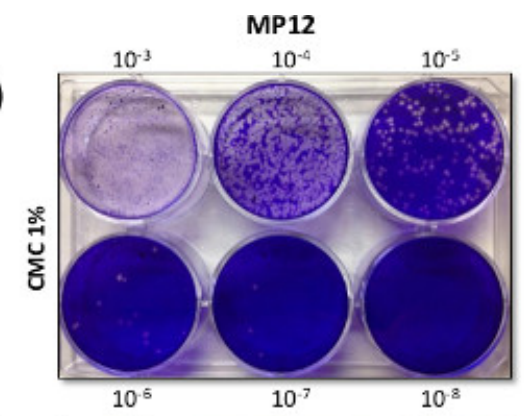

B)

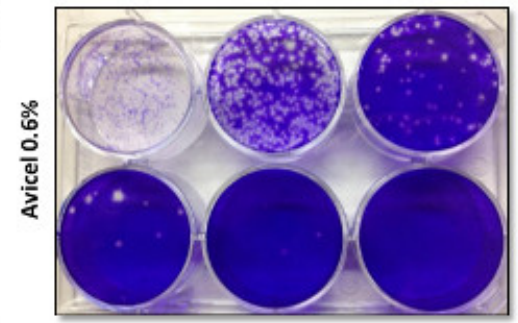

C)

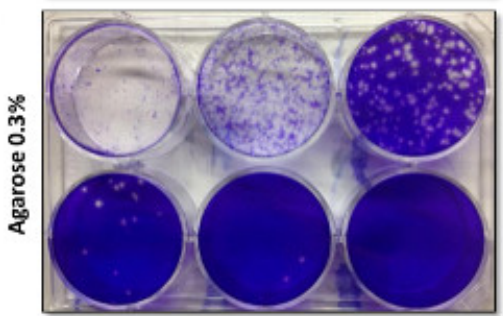

D)

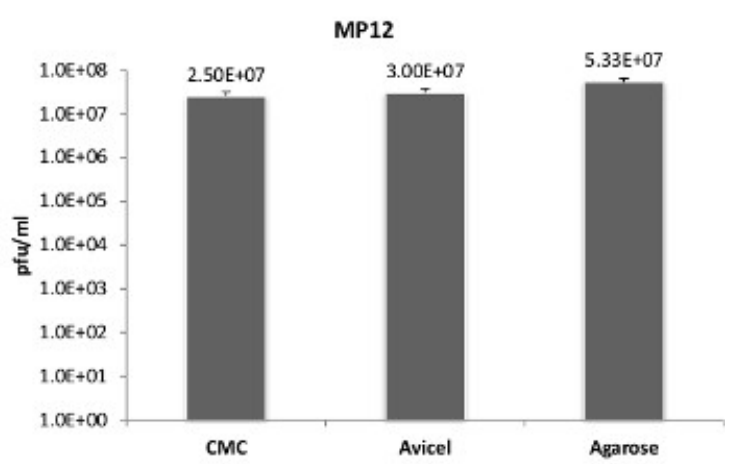

Figure 2: RVFV plaque overlay comparisons utilizing 6 well plates. Veros were plated at $5 \times 10^{5}$ cells in 6 well plates and infected with $400 \mu \mathrm{l}$ using the same serially diluted starting sample of MP12. Three $\mathrm{ml}$ overlays of $0.3 \%$ agarose, $0.6 \%$ Avicel, or $1 \% \mathrm{CMC}$, were applied in order to directly compare the overlays as demonstrated in Panel A, B, and C. Separate experiments were carried out identically as described for Panels A-C, with plaques counted and titered in Panel $D(N=3)$.

A)

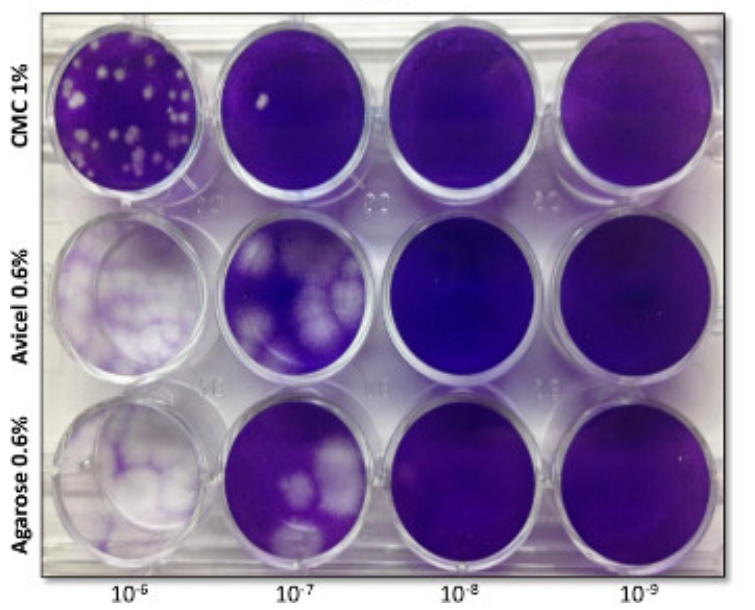

B)

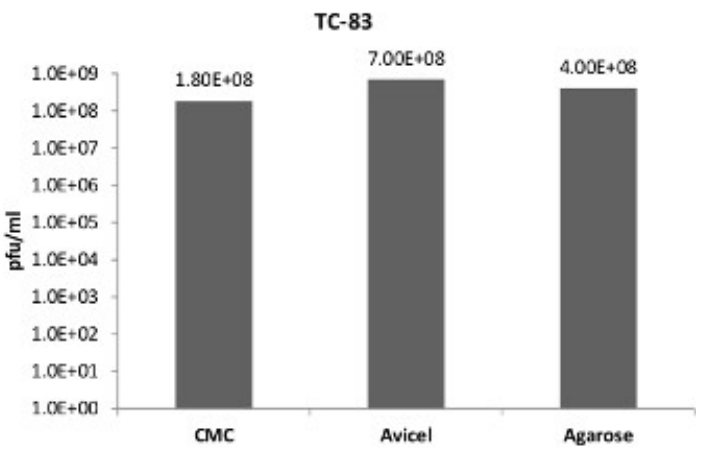

Figure 3: VEEV plaque overlay comparisons utilizing 12 well plates. Veros were plated at $2.5 \times 10^{5}$ cells in 12 well plates and infected with $200 \mu \mathrm{l}$ using the same serially diluted starting sample of the VEEV TC-83 vaccine strain. After infection $1.5 \mathrm{ml}$ overlays of $0.3 \%$ agarose, $0.6 \%$ Avicel, or $1 \%$ CMC, were applied in order to directly compare the overlays as demonstrated in Panel A. Plaques were counted and titered in Panel B. 


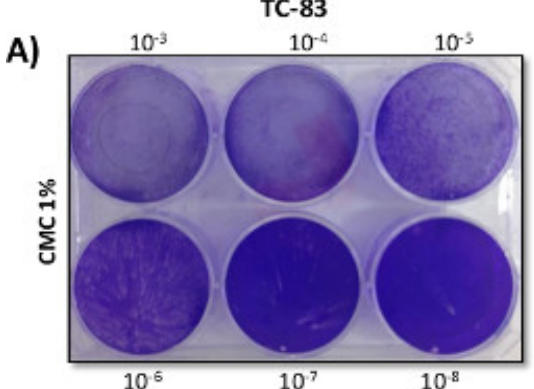

B)

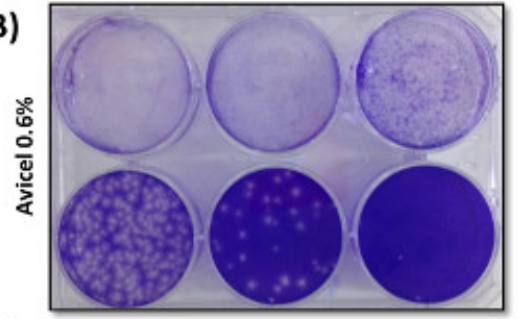

C)

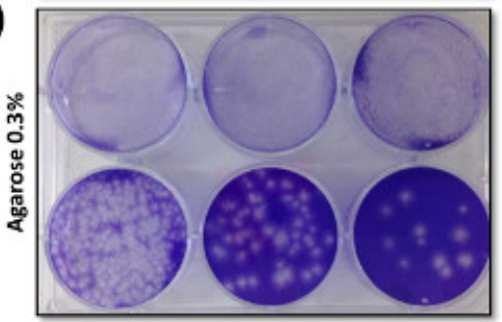

D)

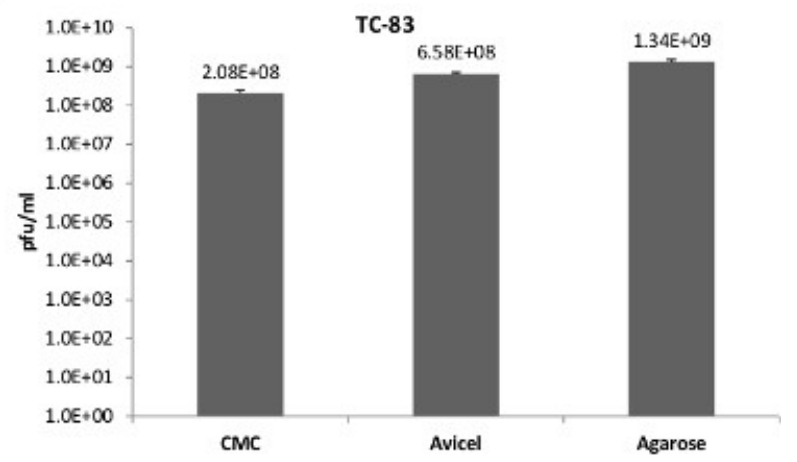

Figure 4: VEEV plaque overlay comparisons utilizing 6 well plates. Veros were plated at $5 \times 10^{5}$ cells in 6 well plates and infected with 400 $\mu \mathrm{l}$ using the same serially diluted starting sample of VEEV TC-83. After infection, $3 \mathrm{ml}$ overlays of $0.3 \%$ agarose, $0.6 \%$ Avicel, or a $1 \%$ CMC, were applied in order to directly compare the overlays as demonstrated in Panels A, B and C. Separate experiments were carried out identically as described for Panels $A$ - C, with plaques counted and titered in Panel $D(N=3)$. 


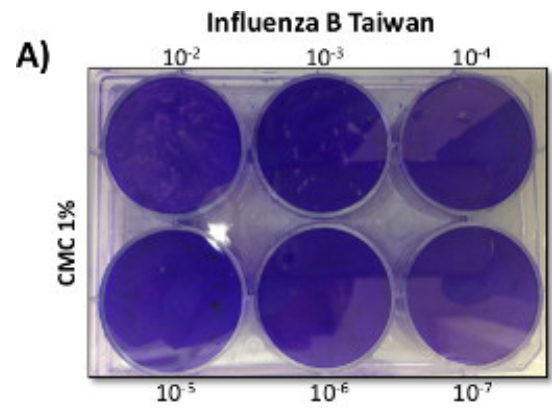

D)

B)

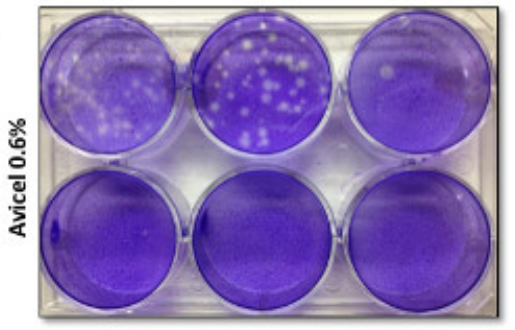

C)
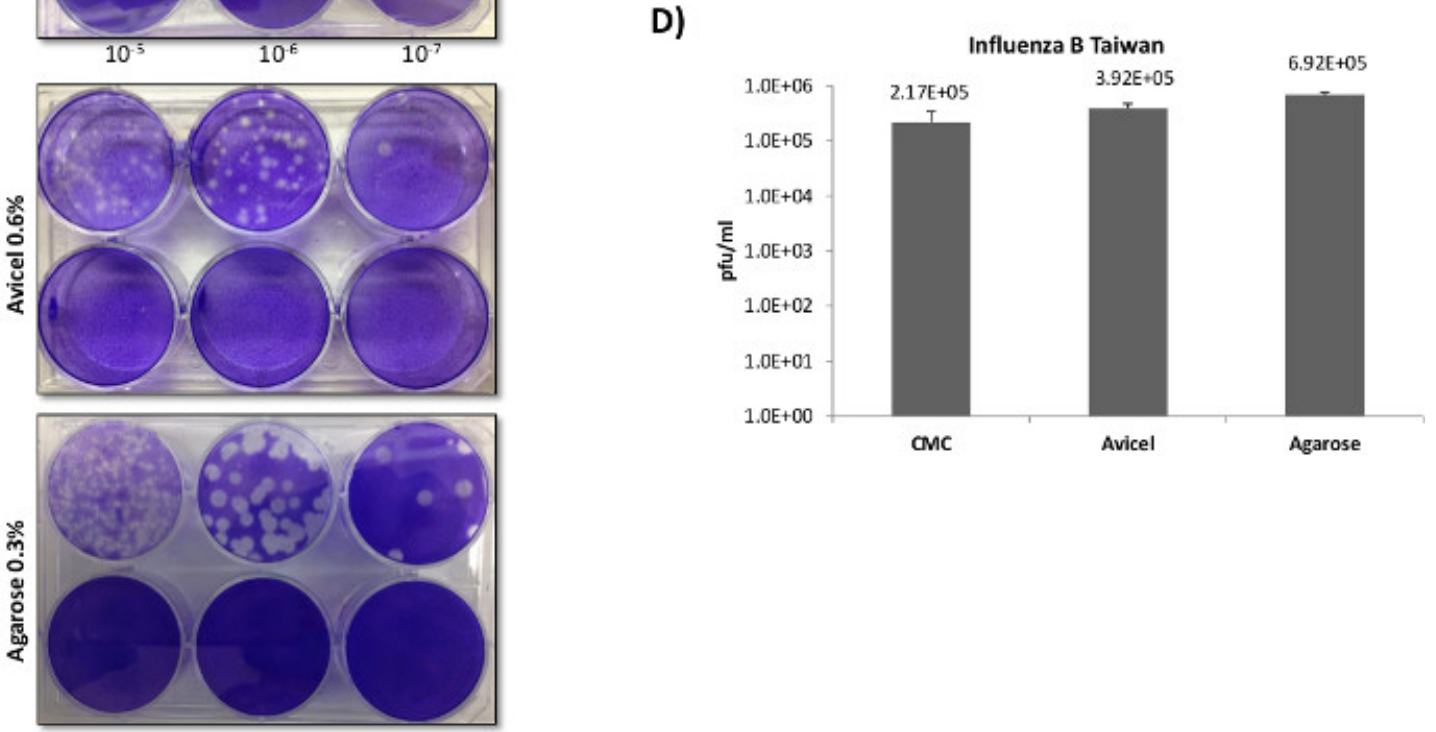

Figure 5: Influenza plaque overlay comparisons. MDCK cells were plated at $5 \times 10^{5}$ cells in 6 well plates and infected with $400 \mu$ l of inoculum using the same serially diluted starting sample of Influenza B Taiwan. No fetal bovine serum (FBS) was used in the growth media or overlays, as FBS can inhibit Influenza propagation through inhibition of certain proteases which are required for viral fusion. TPCK-trypsin was added to all of the overlays prior to application in order to facilitate viral fusion and entry with the host cells. After infection, $3 \mathrm{ml}$ overlays of $0.3 \%$ agarose, $0.6 \%$ Avicel, or $1 \% \mathrm{CMC}$ were applied in order to directly compare the overlays as demonstrated in Panel A, B and C. Separate experiments were carried out identically as described in Panels $A-C$, with plaques counted and titered in Panel $D(N=3)$. While an average was taken for $C M C$ plaques they proved difficult to reliably count as they demonstrated diffuse borders and very small plaque sizes. 


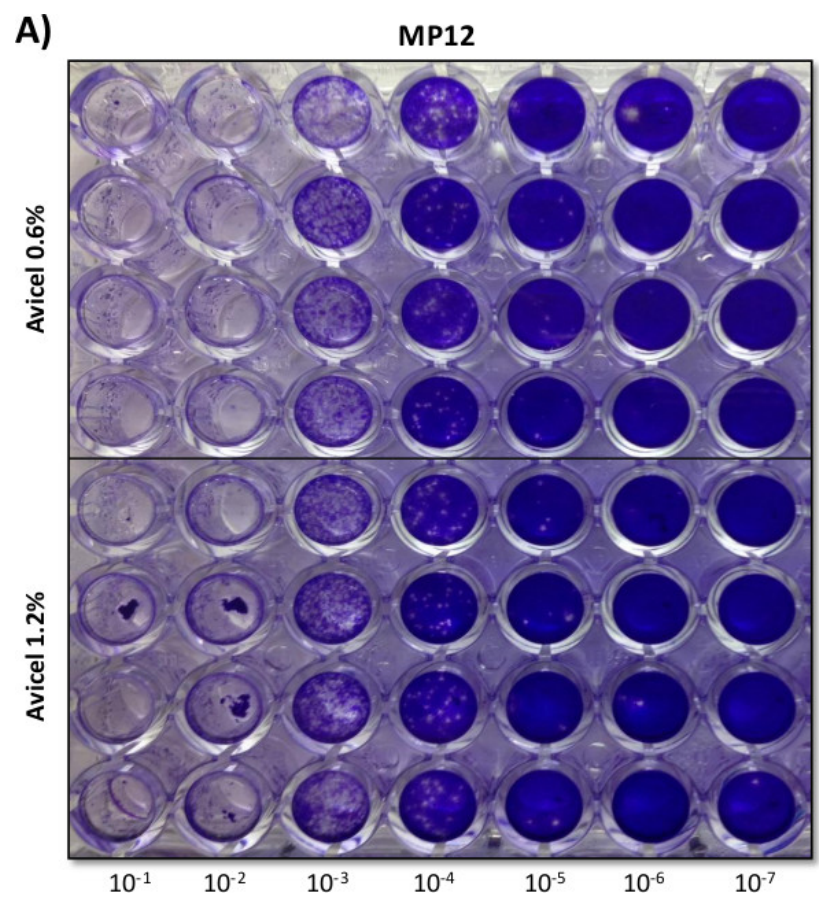

B)

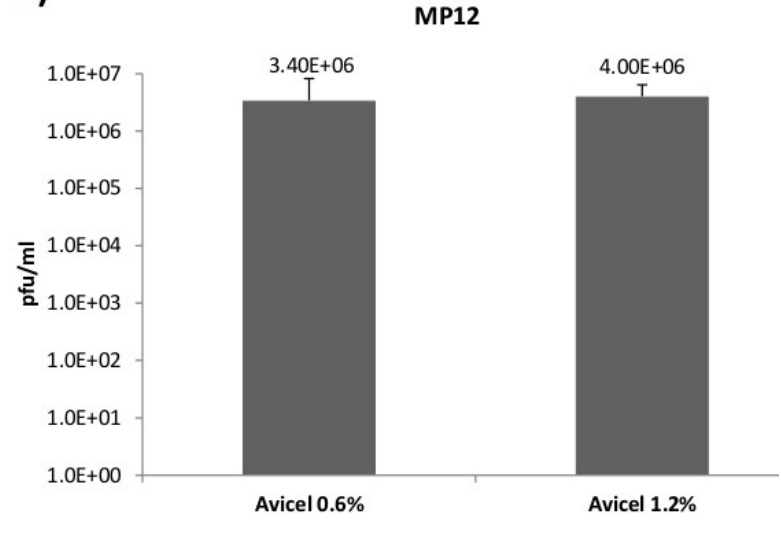

Figure 6: High throughput plaque overlays. A 96 well plate of Veros plated at $3 \times 10^{4}$ cells per well, were infected with $50 \mu$ l of inoculum using the same serially diluted starting sample of RVFV MP12 for $1 \mathrm{hr}$, in quadruplicate. For the overlays, 0.6 and $1.2 \%$ final concentrations of Avicel were trialed in order to determine the feasibility and reproducibility of utilizing liquid overlays in a high-throughput manner, Panels A and B.

\begin{tabular}{|l|l|l|l|}
\hline & RVFV & VEEV & Influenza B \\
\hline Cell type & Vero & Vero & MDCK \\
\hline Infection period & $1 \mathrm{hr}$ & $1 \mathrm{hr}$ & $45 \mathrm{~min}$ \\
\hline Incubation time & 3 days & 2 days & 3 days \\
\hline
\end{tabular}

Table 1: Plaque Assay Inoculation Conditions and Cell Types

\begin{tabular}{|l|l|l|l|}
\hline & RVFV & VEEV & Influenza B \\
\hline Cell Type & Vero & Vero & MDCK \\
\hline Growth Type & DMEM $^{1}$ & DMEM $^{1}$ & DMEM $^{2}$ \\
\hline Plaque Media & 2xEMEM & A & 2xEMEM \\
\hline
\end{tabular}

1. Dulbecco's Modified Eagle Medium supplemented with 10\% Fetal Bovine Serum, 1\% L-Glutamine, 1\% Penicllin/Streptomycin.

2. Dulbecco's Modified Eagle Medium supplemented with 1\% L-Glutamine, 1\% Penicllin/Streptomycin, 0.2\% Bovine Serum Albumin, 0.025\% HEPES, DEAE-Dextran $50 \mathrm{ug} / \mathrm{ml}$.

A. 2x Minimal Essential Media (500 ml) supplemented with 5\% FBS (25 ml), 1\% Minimum Essential Amino Acids (5 ml), $1 \%$ Sodium Pyruvate (5 $\mathrm{ml}), 1 \% \mathrm{~L}$-Glutamine (5 ml), 2\% Pen/Strep (10 ml).

B. $2 x$ Minimal Essential Media $(500 \mathrm{ml})$ supplemented with $0.2 \%$ Bovine Serum Albumin, $1 \%$ Minimum Essential Amino Acids (5 ml), $50 u g / m l$, $0.025 \%$ HEPES, DEAE-Dextran, Trypsin-TPCK*

*Just prior to preparation, add $1 \mu \mathrm{l}$ per $25 \mathrm{ml}$ of $2 \mu \mathrm{g} / \mathrm{ml}$ stock of TPCK-Trypsin to the aliquot you will be using to mix with agarose for plaques.

Table 2: Plaque and Viral/Cellular Growth Medias

\begin{tabular}{|l|l|l|l|}
\hline & RVFV & VEEV & Influenza B \\
\hline Cell type & Vero & Vero & MDCK \\
\hline Infection period & $1 \mathrm{hr}$ & $1 \mathrm{hr}$ & $45 \mathrm{~min}$ \\
\hline Incubation time & 3 days & 2 days & 3 days \\
\hline
\end{tabular}

Overlay solutions will not expire when made so long as sterility is maintained. 
Table 3: Overlays Stock

\begin{tabular}{|l|l|l|l|}
\hline & 6 well & 12 well & 96 well \\
\hline \# of cells/well & $5 \times 10^{5}$ & $2.5 \times 10^{5}$ & $3 \times 10^{4}$ \\
\hline Volume of innoculum $(\mu \mathrm{l})$ & 400 & 200 & 50 \\
\hline volume of overlay $(\mathrm{ml})$ & 3 & 1.5 & 0.100 \\
\hline
\end{tabular}

Table 4: Plate Formats

\section{Discussion}

The most critical factor for a successful plaque assay lies in the optimization of the protocol for the particular viral culture in question as conditions can vary significantly. Key points to take into account are: host cellular compatibility with the virus in question, appropriate viral growth conditions, sufficient dilution ranges in order to clearly differentiate plaques, and correct overlay selection and staining for the cells and virus in question.

While VEEV and RVFV both grow under very similar conditions utilizing many of the same host cell types, differences in plaque morphology and growth kinetics vary significantly. When using Vero cells for plaque assays, which have traditionally been used as a propagation and indicator cell line for hemorrhagic fever viruses and alphaviruses, VEEV typically grows to higher titers than RVFV and demonstrates increased replication kinetics, developing large uniform plaques at $48 \mathrm{hpi}^{4,10-12}$. In contrast to VEEV, RVFV requires $72 \mathrm{hpi}$ and demonstrates plaques that are typically much smaller and variable in size.

In opposition to RVFV and VEEV, the influenza virus is highly host cell specific and can be difficult to propagate through tissue culture. For the influenza virus, viral entry and fusion is normally initiated by the binding of the cell surface receptor with the viral glycoprotein hamagglutinin (HA), which mediates entry into the target cell through binding with the host cell's a-sialic acid surface receptor. HA is a trimeric glycoprotein that is present in the membrane envelope of all Influenza viruses and requires cleavage into the subunits HA1 and HA2 by a specific host cell protease. To further complicate the issue, these cleavage sites can often vary between viral strains ${ }^{13}$. As the expression of proteases capable of cleaving HA is restricted to specific tissues, proteases are often added to cell culture media in order to facilitate viral fusion and entry into the selected host cell ${ }^{13}$. TPCK-trypsin is one example of a commonly used protease that is used in cell culture with both MDCK and Vero cells: facilitating multi-cycle replication (in the absence of any trypsin inactivating serum) through the proteolytic activation of viral $\mathrm{HA}^{14,15}$.

As demonstrated in this study and others, differences in viral life cycles can influence overlay selections, plate formats, and collection times, with significant variances among differing classes and species of viruses. In our study, agarose overlays demonstrated clearer plaques at higher titers than either CMC or liquid overlays for both RVFV and Influenza B virus, reinforcing its utility among a wide range of viruses and cell types. Using a low final concentration of agarose greatly aided in removing the solid plugs and simplified staining. CMC overall demonstrated the poorest efficacy as an overlay for all three viruses tested and produced very small and indistinct plaques for Influenza B virus. Although overall CMC demonstrated the least desirable characteristics, its use in rapidly growing and extremely virulent viruses could prove advantageous, as it did appear to reduce plaque size with only a minimal decrease in titer. The liquid overlay proved to be the most versatile in that it was extremely simple to prepare, increased ease of use, and was comparable to agarose across all of the viruses selected. In a higher throughput 96 -well plate format, application and removal was not inhibited due to solidification as with traditional overlays, and provided accurate and consistent results. A consideration when using liquid overlays lies in the opaque coloring and inability to monitor plaque formation as the plates cannot be moved until the point of collection, limiting this adaptation to viruses with previously characterized replication kinetics.

Minor modifications in plaque assay conditions can significantly alter results, and the evaluation and performance of any new system or technique is always warranted. While an optimized and standardized plaque assay protocol does not exist for every situation, our report demonstrate the versatility and ease of use in traditional as well as novel liquid overlays for plaque assays, while providing a sufficient background for further user modifications.

\section{Disclosures}

The authors have nothing to disclose and no competing or financial interests in the protocol or products described.

\section{Acknowledgements}

We would like to thank FMC BioPolymer USA for product samples of Avicel. This work was supported through the Defense Threat Reduction Agency grant HDTRA1-13-1-0005 to KK and the NIH research grant 1R15AI100001-01A1to KK.

\section{References}

1. Cooper, P. D. The plaque assay of animal viruses. Adv. Virus Res. 8, 319-378 (1961)

2. Dulbecco, R. \& Vogt, M. Some problems of animal virology as studied by the plaque technique. Cold Spring Harb. Symp. Quant. Biol. 18, 273-279 (1953)

3. Hartley, J. W. \& Rowe, W. P. Tissue culture cytopathic and plaque assays for mouse hepatitis viruses. Proc. Soc. Exp. Biol. Med. Soc. Exp. Biol. Med. N. Y. 113, 403-406 (1963). 
4. Juarez, D., Long, K. C., Aguilar, P., Kochel, T. J. \& Halsey, E. S. Assessment of plaque assay methods for alphaviruses. J. Virol. Methods. 187, 185-189, doi: 10.1016/j.jviromet.2012.09.026 (2013).

5. Shurtleff, A., Keuhne, A., Biggins. J., \& Keeney, A. Use of alternative overlays in plaque assays. USAMRIID Fort Detrick MD USA Unpublished work (2011).

6. Shurtleff, A. C. et al. Standardization of the Filovirus Plaque Assay for Use in Preclinical Studies. Viruses. 4, 3511-3530, doi: 10.3390/ v4123511 (2012).

7. Matrosovich, M., Matrosovich, T., Garten, W. \& Klenk, H.-D. New low-viscosity overlay medium for viral plaque assays. Virol. J. 3, 63, doi: 10.1186/1743-422X-3-63 (2006).

8. Klebe, R. J. \& Harriss, J. V. A technically simple 'non-lethal' vital staining procedure for viral plaque and cell transformation assays. Arch. Virol. 81, 359-362 (1984).

9. Gaush, C. R. \& Smith, T. F. Replication and plaque assay of influenza virus in an established line of canine kidney cells. Appl. Microbiol. 16, 588-594 (1968).

10. Abiko, C. et al. Outbreak of Human Metapneumovirus Detected by Use of the Vero E6 Cell Line in Isolates Collected in Yamagata, Japan, in 2004 and 2005. J. Clin. Microbiol. 45, 1912-1919, doi: 10.1128/JCM.01251-06 (2007).

11. Baer, A. et al. Induction of DNA damage signaling upon Rift Valley fever virus infection results in cell cycle arrest and increased viral replication. J. Biol. Chem. 287, 7399-7410, doi: 10.1074/jbc.M111.296608 (2012)

12. Austin, D. et al. p53 Activation following Rift Valley Fever Virus Infection Contributes to Cell Death and Viral Production. PLoS ONE. 7, doi: 10.1371/journal.pone.0036327 (2012)

13. Böttcher-Friebertshäuser, E., Stein, D. A., Klenk, H.-D. \& Garten, W. Inhibition of influenza virus infection in human airway cell cultures by an antisense peptide-conjugated morpholino oligomer targeting the hemagglutinin-activating protease TMPRSS2. J. Virol. 85, 1554-1562, doi: 10.1128/JVI.01294-10 (2011).

14. Youil, R. et al. Comparative study of influenza virus replication in Vero and MDCK cell lines. J. Virol. Methods. 120, 23-31, doi: http:/l dx.doi.org/10.1016/j.jviromet.2004.03.011 (2004).

15. Tobita, K., Sugiura, A., Enomoto, C. \& Furuyama, M. Plaque assay and primary isolation of influenza a viruses in an established line of canine kidney cells (MDCK) in the presence of trypsin. Med. Microbiol. Immunol. (Berl.). 162, 9-14, doi: 10.1007/BF02123572 (1975). 